\title{
Corner Detection Using Color Density Gradient
}

\author{
Sun $\mathrm{Da}^{1}$ Huang Jianhua ${ }^{1}$ Tang XiangLong ${ }^{1}$ \\ ${ }^{1}$ School of Computer Science and Technology, \\ Harbin Institute of Technology, Harbin China
}

\begin{abstract}
A novel corner detection approach which is based on clustering the pixels by density gradient is proposed. Moving along the density gradient, the pixels will arrive at their object center, and at corner neighborhood, the density gradients all point away in any direction. Hence, two eigenvalues of the Hessian matrix are all positive and significant in density gradient field. The proposed method is invariant to the detection scale, and it can be applied for all type images.
\end{abstract}

Keywords: Corner detection; Density gradient; Color corner

\section{Introduction}

Corner is one of important low-level image features, and it is widely applied in image analysis, alignment and retrieval. On image plane, the corners are some steady point, at which the intensity changes greatly in any direction. Therefore, most methods use the intensity gradient to describe the intensity variance, and find the corners in the gradient field. In the intensity gradient based methods, Harris corner detector [1] is the most popular one which uses the second moment matrix to capture the local structure of the corner, and it has been extended to scale and affine invariant detector by selecting the scale parameters [2]. While estimating the intensity or color gradient in large scale, the mean filter is used to smoothing the image; as a result, the corner is rounded and the position of the corner will be shifted with detection scale increasing. Furthermore, the 'mean color' may distort the original color information, and then affect the detection results [3].

In this paper, a novel detector which finds the corners in density gradient field is present. In the density gradient field of image, the density gradients point away from the edge along the direction perpendicular to the edge, and around a corner, the density gradients are all point away in all directions. Therefore, the corners are source points in the density gradient field, and the Hessian matrix of the density has two significant positive eigenvalues at corners. As the edges are preserved while density estimation, the corners located in the density gradient filed are invariant to the detection scale. And the density estimation is uniform for all type images, so the new detector can be fit for color image.

\section{Density based corner detector}

A digital image can be represented as a two-dimensional lattice of the feature vectors, and the feature vector dimension $d_{c}=1$ for gray-level image, $d_{c}=3$ for color image, and $d_{c}>3$ for multispectral image [4]. With joining the image plane and feature space, the pixels are just some sample points distributed in a spatialfeature space with dimension $d_{c}+2$. At

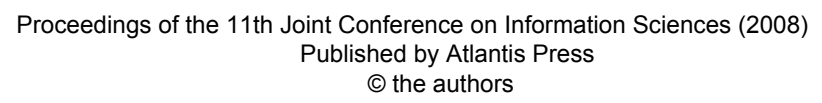


pixel $\mathrm{m}(x, y, c)$, the density and density gradient on image plane is estimated by

$$
\begin{gathered}
D(m)=\frac{C}{h_{s} h_{f}} \sum_{w} K\left(\frac{\left\|x-x_{i}, y-y_{i}\right\|}{h_{s}}\right) K\left(\frac{\left\|c-c_{i}\right\|}{h_{f}}\right) \\
\nabla_{s} D(m)=\left(D_{x}, D_{y}\right)
\end{gathered}
$$

where, $K(x)$ is the kernel function, $C$ is normalized constant, $\left(x_{i}, y_{i}, c_{i}\right)$ is the pixel in the estimator window $w, h_{s}$ is spatial bandwidth in image plane and $h_{f}$ is feature bandwidth in feature space.

In the clustering based image segmentation technique, the pixels in the same object region will be put into one class, and the edges are just the decision boundary used to classify the pixels. The density gradient is one of important clustering information, and the pixels can arrive at nearest mode along their density gradient [4]. On opposite sides of edge, the pixels belong to different classes, so that the density gradient perpendicular to the edge and points away. Furthermore, around a corner, the density gradients point away in all directions, as Fig. 1 (a) shows.

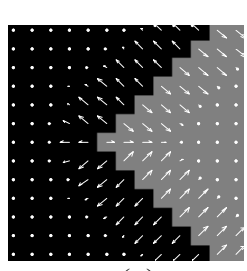

(a)

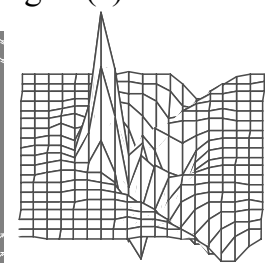

(b)
Fig.1. (a) Density gradients of the pixels around a corner. (b) The corner response at in density gradient field of (a).

As the pixels are moving along density gradient while clustering, the motion trajectory of the pixels can be described by first order differential equations:

$$
\left\{\begin{array}{l}
\frac{d x}{d t}=D_{x}=\frac{\partial D_{x}}{\partial x} x+\frac{\partial D_{x}}{\partial y} y \\
\frac{d y}{d t}=D_{y}=\frac{\partial D_{y}}{\partial x} x+\frac{\partial D_{y}}{\partial y} y
\end{array}\right.
$$

The coefficient matrix $M$ can describe the distribution of density gradients in a neighborhood.

$$
M=\left[\begin{array}{ll}
\frac{\partial D_{x}}{\partial x} & \frac{\partial D_{x}}{\partial y} \\
\frac{\partial D_{y}}{\partial x} & \frac{\partial D_{y}}{\partial y}
\end{array}\right]
$$

Three cases need to be considered:

(1) If the neighborhood is on one side of edge, the pixels belong to same class, and then they moving in the same direction. Therefore, the eigenvalues of $M$ are negative or small.

(2) If the neighborhood is on the edge, the pixels are all moving away perpendicular to the edge. Therefore, one eigenvalue of $M$ is positive, and the other is small.

(3) If the neighborhood is on a corner, the pixels are moving away in all directions.

Therefore, the eigenvalues of the matrix $M$ are all positive and significant. Because, $M$ have two positive eigenvalues, that is equal to $\operatorname{Det}(M)>0$ and $\operatorname{Tr}(M)>0$. Harris proposed a measure function:

$$
R=\operatorname{Det}(M)-k \operatorname{Tr}(M)^{2}
$$

where, $k$ is a coefficient and usually $k=0.04$. The response $R$ is great positive, i.f.f., $\lambda_{1}, \lambda_{2}$ are all significant. $\operatorname{Det}(M)$ must be positive while $R$ is positive, and the $\operatorname{Tr}(M)=D_{x x}+D_{y y}$ is just the divergence in the density gradient field. So, at corner, the divergence and $R$ are all positive, and like Harris corner detector, $R$ is taken as the corner response, which is local maximum at corner.

In summary, the process of corner detection is

(1) Estimate the density gradient at each image point.

(2) Compute the divergence and corner response at each point.

(3) Label the corners at the points with positive divergence and local positive maximum corner response. 
Fig. 2 shows the 100 corners detected by the density gradient based detector (DGBD). If only estimate a special color's density at every image point using Eq.(1), and then the color's corners can be located by the new detector. As Fig.3 show only the corners with the color of the building are found by DGBD.

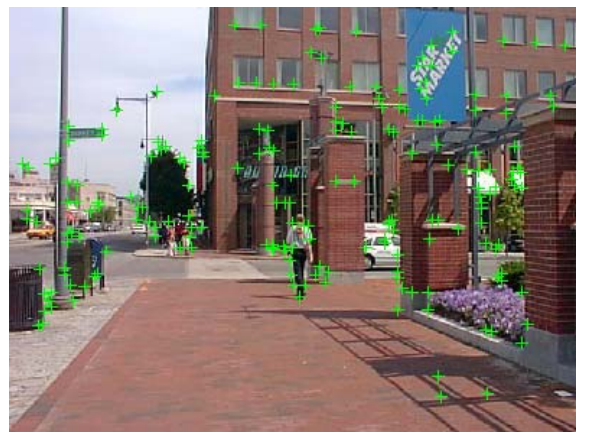

Fig.2 The corners detected by the DGBD in color image.

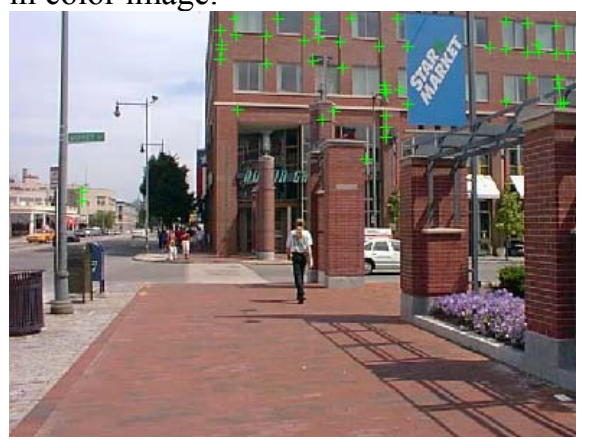

Fig.3 The corners with the color of the building detected by DGBD.

\section{Evaluation}

In large detection scale, the noised and textures will be filter out, and the important objected information are enhanced. Therefore, we are more concerned about the corners detection in large scale. The density gradient based detector can preserve the corner position while density estimation scale increasing, as Fig.2 (a) shows, because the density estimation is realized by smoothing in the joint spatialfeature space, and Darin [4] illuminated that the edges are preserved while density gradient estimation. But the intensity gradient is estimated by smoothing the intensity function of the image, and the corner point in intensity gradient field will change with the smoothing scale increasing, as Fig.4 (b) shows.

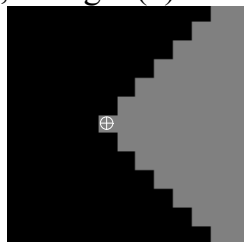

(a)

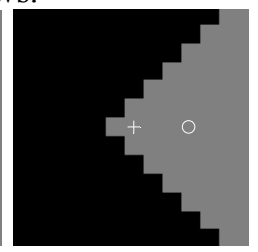

(b)
Fig.4. (a) The corner points located in density gradient based detector. (b) Corners located by intensity gradient based detector. For two detectors, ' + ' is with the detection scale $\sigma=1$, and ' $\mathrm{o}$ ' is with the detection scale $\sigma=4$.

In new corner detection method, the density gradient is used to take place of the intensity gradient while compute the Harris corner response. Because Harris corners are invariant to the rotation change, the scale stability of density gradient based method is compared with that of the intensity gradient based method. In [18] Schmid uses the repeatability as the criterion to evaluate the interest point detectors and it is widely adapted to evaluate the new detectors. The repeatability score defined by Schmid [18] is the ratio between the number of correspondence points and the smaller number of points detected in one of the images. The set of correspondence points $R_{i}(\varepsilon)$ and the new repeatability $r_{i}(\varepsilon)$ is defined as (6) and (7): $R_{i}(\varepsilon)=\left\{\left(\tilde{x}_{1}, \tilde{x}_{i}\right) \mid \operatorname{dist}\left(H_{1 i} \tilde{x}_{1}, \tilde{x}_{i}\right)<\varepsilon\right\}$

$r_{i}(\varepsilon)=\frac{\left|R_{i}(\varepsilon)\right|}{\min \left(n_{1}, n_{i}\right)}$

where, $\mathrm{H}_{1 \mathrm{i}}$ is the homograph between images $I_{l}$ and $I_{i .} \varepsilon$ is the max distance of two 
correspond points, $n_{l}, n_{i}$ are the number of the points in each image. The boat images from Mikolajaczyk's [2] evaluation sequences(http://www.robots.ox.ac.uk/ vgg /research/affine/index.html) are used. For each detector, the gradients are estimated in scales $\sigma=1$ and 4 on image plane, and the feature scale parameter of the density gradient estimation is $h_{f}=128$. On each image, 200 corner points are found, and the repeatability of the detectors are shown in Fig. 5. In small detection scale $(\sigma=1)$, the stability of two detectors are similar, but in large detection scale $(\sigma=$ 4 ), the scale stability of density gradient based method is distinctly better than that of intensity gradient based method.

\section{Conclusions}

The corners detected in the density gradient field are invariant to the detection scale. Therefore the new detector can enhance the large scale large scale corners, and the stability is better than the intensity gradient detector. Additionally, the new method is also fit for color image.

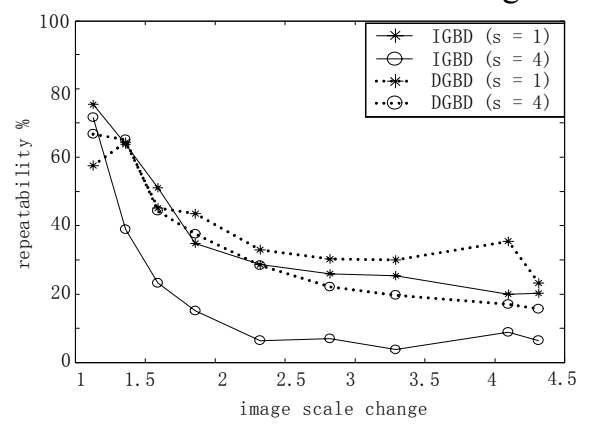

Fig.5. Repeatability of the intensity gradient based detector (IGBD) and density gradient based detector (DGBD) with respect to image scale changes.

\section{References}

[1] C. Harris, M. Stephens, A Combined Corner and Edge Detector. Proceedings of The Fourth Alvey Vision
Conference, Manchester, pp 147-151. 1988

[2] K.Mikolajczyk, C.Schmid, Scale \& Affine Invariant Interest Point Detectors. International Journal of Computer Vision, 60(1):63-86, 2004.

[3] Mark A. Ruzon, Carlo Tomasi. Edge, Junction, and Corner Detection Using Color Distributions. IEEE Transactions on Pattern Analysis and machine intelligence, 23(11): 12811295,2001

[4] D. Comaniciu, P. Meer, Mean Shift: A Robust Approach Toward Feature Space Analysis. IEEE Transactions on Pattern Analysis and machine intelligence, 24(5):603-609, 2002 